УДК 336.761 .6

\author{
Чикатуева Диана Эдуардовна, Сильченко Алина Сергеевна, \\ Рачкова Татьяна Геннадиевна, Оробец Кирилл Геннадьевич
}

\title{
ФРАКТАЛЬНОЕ ПРОГНОЗИРОВАНИЕ ФИНАНСОВЫХ РЫНКОВ
}

Данная статья посвящена использованию фрактального метода на финансовом рынке. Описывается краткая история появления термина «фрактал», использование фрактального метода и его свойств. Приведен перечень преимуществ и возможностей данного метода на межсународном финансовом рынке Форекс. Произведен обзор факторов, а также степень их влияния на фрактальный анализ. Доказаны фрактальные связи между ценами на биржах и повторялоцимися структурами посредством использования программ компьютерного моделирования. Были рассмотрены торговые стратегии и особенности их использования.

Ключевые слова: финансовые рынки, Форекс, методы прогнозирования, фрактал, фрактальный метод.

\section{Diana Chikatueva, Alina Silchenko, Tatiana Rachkova, Kirill Orobets} FRACTAL FORECASTING FINANCIAL MARKET FOREX

This article focuses on the use of the fractal method in the financial market. Describes a brief history of the emergence of the term "fractal", the use of the fractal method and its properties. The list of advantages and opportunities of this method in the international financial market Forex is given. A review of the factors, as well as the degree of their influence on the fractal analysis. Proven fractal relationships between prices on exchanges and repetitive structures through the use of computer simulation programs. Were considered trading strategies and features of their use.

Key words: financial markets, Forex, forecasting methods, fractal, fractal method.

Bведение / Introduction. Сегодня финансовые рынки вызывают большой общественный интерес. Финансовой аналитикой занимаются все, кто работает для глобальных корпораций и государственных учреждений (трейдеры, аналитики и т. д.). Существует множество способов анализа событий, происходящих на рынках. Они включают:

1) технический анализ;

2) фундаментальный анализ;

3) волновой принцип Эллиотта и многие другие менее известные методы.

Однако следует выделить метод, который занимает уникальное место среди всех остальных благодаря своей простоте и оригинальности. Этот метод называется фрактальным анализом.

Фрактальное прогнозирование финансового рынка Форекс / Fractal forecasting financial market Forex. Термин «фрактал» был введен французским экономистом и математиком Бенуа Мандельбротом в 1975 году. Именно он считается создателем фрактальной геометрии. Бенуа Мандельброт был первым человеком, который обратил внимание на повторяющееся ценообразование. Анализ цен на хлопок более чем за столетний период привел к созданию эффективной торговой методики, впервые упомянутой в известном «торговом хаосе» Биллом Уильямсом. Именно Уильямс впервые предположил, что рынок является хаотичным, многофакторным и саморегулирующимся. В связи с этим он сделал вывод об отсутствии необходимости использования линейных моделей. 
Применение фрактального метода в торговле активно используется крупными участниками рынка и является лучшим показателем надежности фракталов. Комбинации фракталов обладают такими свойствами, как:

1) нерегулярность: в случае если фрактал описывается функцией, то такая функция будет недифференцируемой. Это отражает тот факт, что порой цены на рынке могут обладать высокой волатильностью. Поэтому задачей трейдеров является наведение порядка и минимизация хаоса на рынке;

2) самоподобие: данное свойство означает, что каждая часть фрактала очень схожа как с другими его частями, так и с самим фракталом в целом;

3) размерность: наличие различной размерности фракталов на кривой позволяет определять и прогнозировать нестабильные состояния системы.

Таким образом, рассмотрев все вышеизложенные свойства, можно сделать вывод, что фракталы могут быть успешно использованы для составления ценовых прогнозов.

Этот фактор является причиной использования фрактального метода на рынке Форекс.

Наличие фрактальных связей между ценами на биржах и повторяющимися структурами доказано посредством использования программ компьютерного моделирования.

Фрактал означает устойчивую масштабируемую конструкцию неправильной формы, возникающую для любых данных. Если брать в рассмотрение рынок финансов, то фрактал в нем представлен в виде свечного паттерна. Данный паттерн имеет от пяти и более свечей, при этом максимум или минимум центральной свечи должен быть больше экстремумов ближайших свечей. На рис. 1 представлена классическая схема фрактальной конструкции.
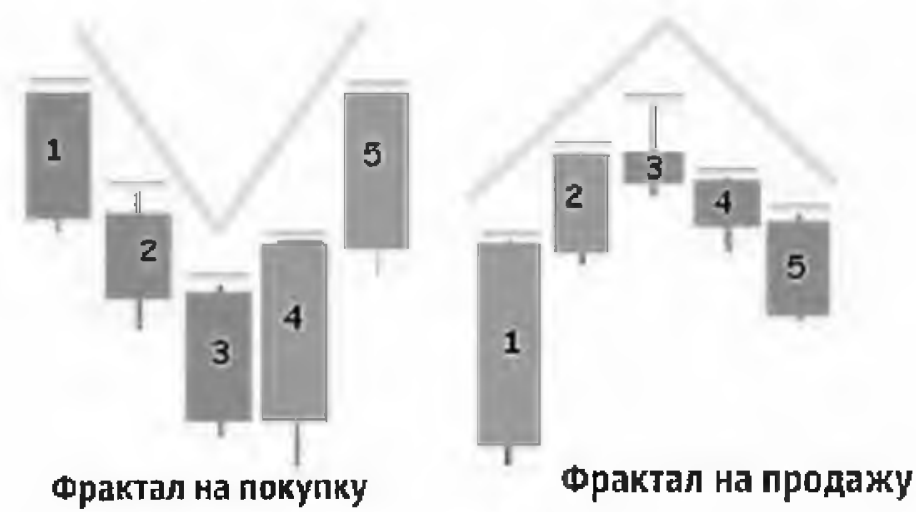

Рис. 1. Классическая схема фрактальной конструкции

Возникновение фракталов предопределяет начало новой ценовой пирамиды и трактуются как рекомендации трейдерам.

Фрактал на покупку представлен в виде пяти или более свечей, которые расположены последовательно и образовывают паттерн, где экстремум максимума определяет свеча, находящаяся посередине.

Фрактал на продажу является полной противоположностью фрактала на покупку. Он, аналогично предыдущему паттерну, состоит из пяти или более последовательных баров, но средняя свеча в этом случае указывает на экстремум минимума.

Данный анализ направлен на своевременное выявление и обработку фрактальных конструкций совместно с иными показателями рынка независимо от их характеристик. Множество реальных фракталов является очень динамичным: оно может меняться в зависимости от количе- 
ства и структуры. Если брать в рассмотрение появление на фрактале точек максимума и минимума, то они не обязательно должны чередоваться. Основным требованием должно быть наличие явного экстрима. Поэтому на практике часто можно встретить использование нестандартных комбинаций, примеры которых представлены на рис. 2.

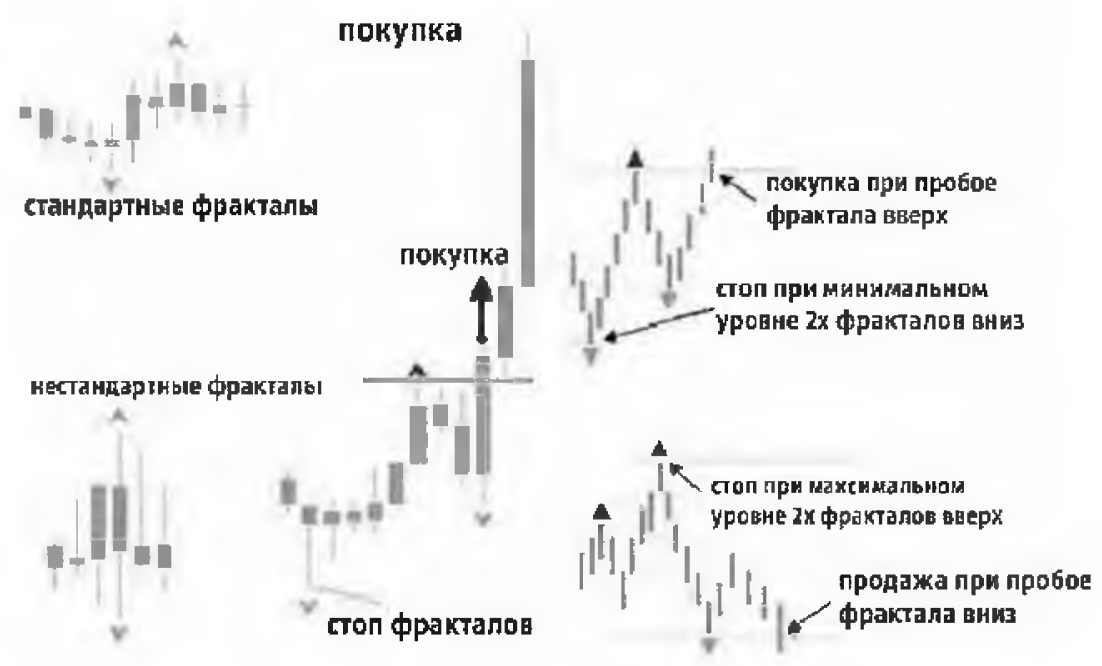

Рис 2. Нестандартные фрактальные комбинации

При появлении точек максимума или минимума на нескольких свечах паттерна в качестве рекомендаций трейдерам следует учитывать только последнюю точку.

Если изменение цены выше уровня фрактала на покупку, то это прорыв продавцов, в случае если изменение цены ниже фрактала на продажу, то это прорыв покупателей. Примерная схема приведена также на рис. 2.

Появление фрактала обозначает значительные изменения в движении при формировании паттерна. Самый сильный сигнал появляется сразу после его появления, и одну и ту же группу свечей можно использовать как для верхних, так и для нижних фракталов. Указатели появившихся фракталов не перерисовываются.

Экстремумом максимума будет являться граница верхнего фрактала, аналогично, экстремумом минимума - граница нижнего фрактала. При таких условиях в торговле с использованием фракталов направление входа должно быть противоположным созданному фракталу, точка остановки на 1-5 пунктов выше или ниже границы ключевой свечи. Если же происходит образование фрактала, противоположного данному, то торговый сигнал будет отменен.

Если возникают фракталы разных направленностей, которые расположены близко друг к другу, а иногда и на одном баре, то в данном случае речь идет о фрактальном старте. Тогда финансовый рынок должен будет выбрать одно из направлений фракталов.

Выполнение фрактального анализа предполагает учет следующих типичных факторов:

1) длительность образования фрактальной структуры прямо пропорциональна надежности торговых сигналов трейдерам;

2) увеличение количества 《ложных» фракталов и пробоев определяется уменьшением торгового периода. Длительное ожидание создания «истинного» фрактала является причиной пропуска оптимальной точки входа.

Если на каком-то уровне создаются несколько фракталов разной направленности с последующим пробоем уровня, то целесообразно ожидать сильного и длительного тренда в направлении пробоя. 
В импульсном движении начало или конец могут быть определены фракталом, который, в свою очередь, представляется как волновая структура. Использование фракталов в волновом анализе возможно только на периодах от D и выше. В случае определения меры соответствия методик исследования и их результатов необходимо правильно проанализировать структуру бара, на которой этот фрактал был образован.

Если пробойная свеча закрывается за пределами уровня накопления, то ее можно открывать в направлении пробоя на меньшем торговом периоде.

Если конструкция свечи является поворотной моделью, то вероятность появления обратного фрактала и перехода в режим времени, при котором цена не понижается и не падает, повышается.

Подлинный пробой фрактала на любом торговом периоде должен обосновываться определенными объемами. Твердый пробойный бар, но с малым объемом, на первый взгляд считается нестабильным сигналом. Помимо этого, для оценки объемов рекомендуется использование стандартных конструкций ценового действия.

На сегодняшний день поиск фракталов на графике цен осуществляется не вручную, а при помощи специальных пакетов торговых платформ с наличием в них индикатора Fractals. C помощью этого индикатора можно выделить две торговых стратегии.

Для использования данной стратегии необходимы регулярные фракталы и индикаторы аллигатора со стандартными настройками (рис. 3). Мы торгуем всеми основными валютными и фьючерсными активами: для анализа и входа-M15, для сопровождения - от Н1 выше (для фьючерсов - от D1 выше).

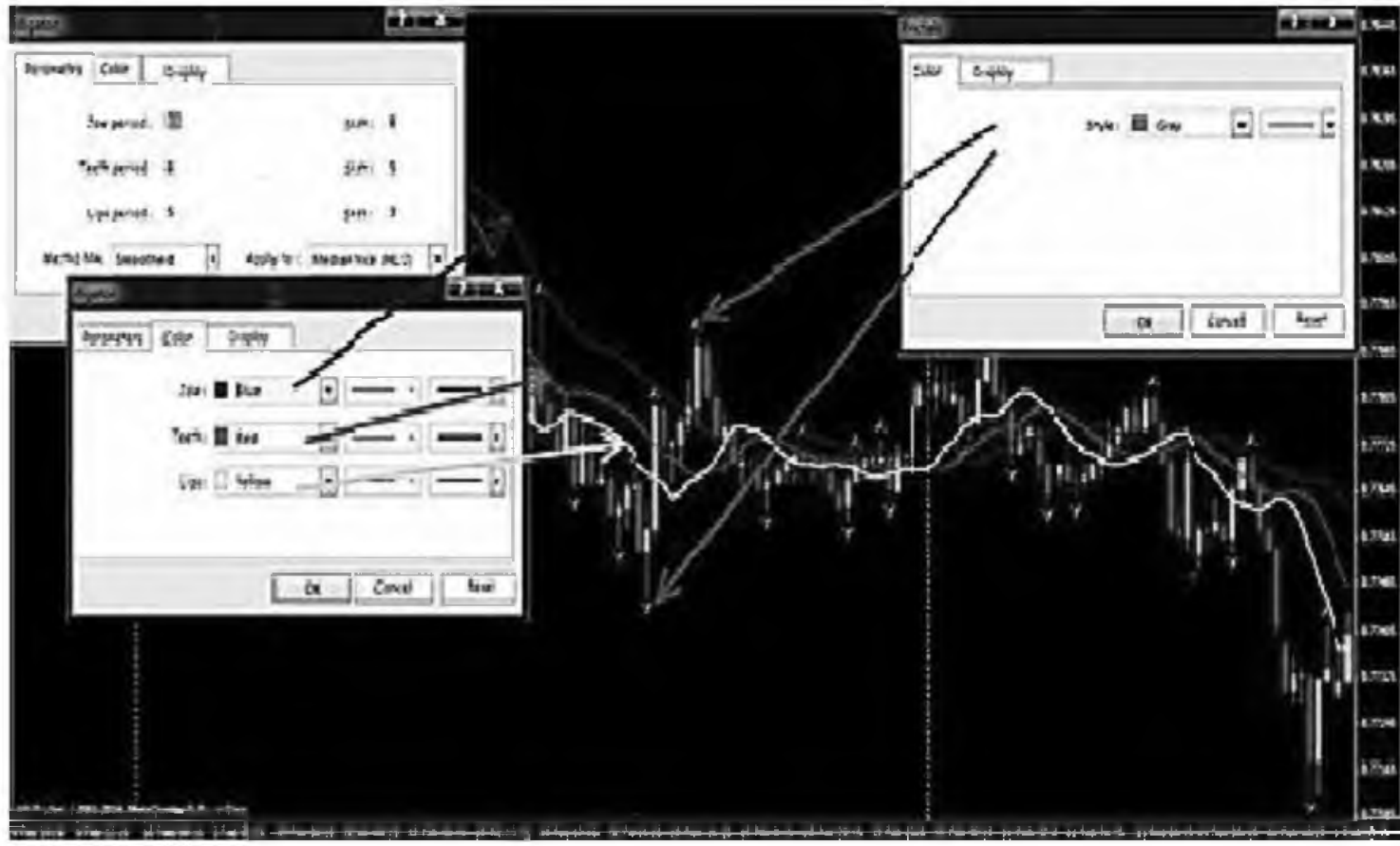

Рис. 3. Стратегия Уильямс «Alligator и Fractals»

Схема входа для фракталов Форекс показана на рис. 4. Биржевые заявки трейдеров находятся на обратном экстремуме от двух последних фракталов: покупка ниже самого низкого, продажа немного выше самого высокого. Удерживание сделки осуществляется до формирования противоположного фрактала. Во том промежутке времени, когда цена не меняется, мы игнорируем все фракталы. 


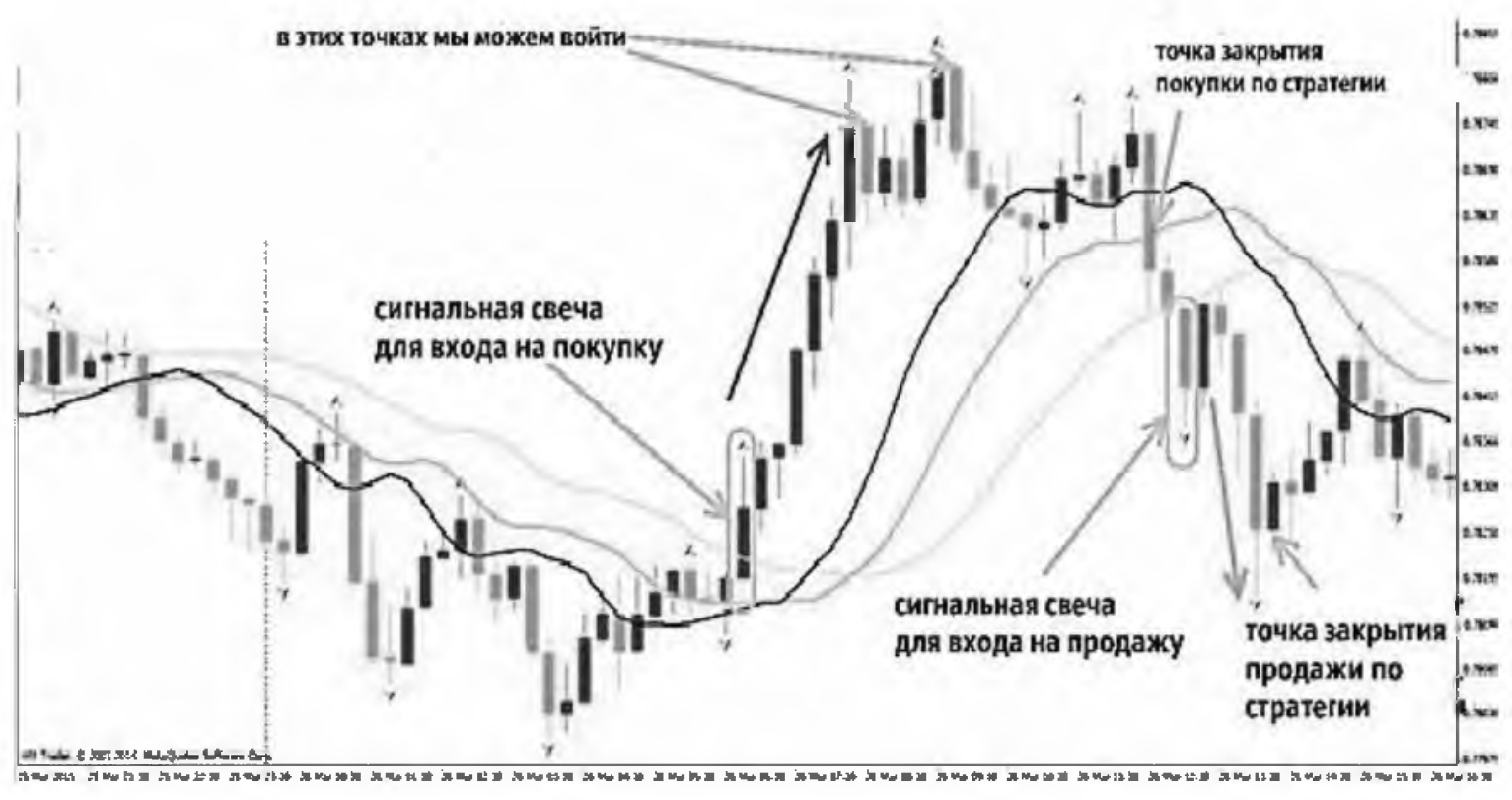

Рис. 4. Схема входа для фракталов Форекс

Данная методика осуществления фрактального анализа предполагает учет следующих факторов:

1) торговля должна реализовываться только по направлению аллигатора;

2) первый фрактал является предупреждающим сигналом после выхода аллигатора;

3) при длинном и узком периоде времени, когда цена не повышается и не понижается, можно ставить отложенные ордера за границы небольших фракталов;

4) красная линия аллигатора является линией динамического стопа;

5) следующий вход может осуществиться только после закрытия стопа и появления нового фрактала.

Торгуется любой валютный актив средней волатильности, подверженный устойчивым тенденциям: EUR / USD, AUDUSD, GBP / USD. D1 является периодом для анализа и ввода. Как правило, стопы и прибыли обычно не ставятся, в конце они закрываются вручную.

Наличие фрактала вверх обозначает продажу, а наличие фрактала вниз - покупку. Вход будет осуществляться не только по рыночному ордеру, но и по отложенным ордерам:

1) покупка - BuyStop на 2-5 пунктов выше максимума второй свечи, следующей за ключевой свечой фрактала;

2) продажа - SellStop на 2-5 пунктов ниже минимума второй свечи, следующей за ключевой.

Заключение / Conclusion. Использование фрактальной торговли возможно лишь во время стабильного тренда. Тогда она является наиболее эффективной. Но при длительном периоде времени, когда цена не повышается и не понижается, применение фрактального метода может оказаться убыточным. Следует брать в учет тот факт, что первоначально торговая стратегия фракталов была разработана для фондового рынка, который отличался меньшей волатильностью и был более прогнозируемым. Непредвиденные ситуации на рынке Форекс часто являются причинами ошибок в расчете построения фракталов, а также возникновения «ложных» или трудных для распознавания сигналов. Однако взаимодействие фракталов и трендовых инструментов является мощным инструментом технического анализа и может стать основой построения стабильно прибыльной системы. 


\section{ЛИТЕРАТУРА И ИНТЕРНЕТ-РЕСУРСЫ}

1. Мандельброт Б. Фрактальная геометрия природы. Сан-Франциско: W. H. Freeman, 1982.

2. Петерс Э. Фрактальный анализ финансовых рынков. Применение теории хаоса в инвестициях и экономике. М.: Интернет-трейдинг, 2004. 304 с.

3. Мандельброт Б., Хадсон Р. Л. (Не)послушные рынки. Фрактальная революция в финансах. М.: Вильямс, 2006. 408 с.

4. Петерс Э. Хаос и порядок на рынках капитала. М.: Мир, 2000. 333 с.

5. Алмазов А.А. Фрактальная теория рынка Forex. M.: AdmiralMarkets, 2009. 296 c.

\section{REFERENCES AND INTERNET RESUORCES}

1. Mandelbrot B. Fraktal'naya geometriya prirody (The Fractal Geometry of Nature). San Francisco: W. H. Freeman, 1982.

2. Peters E. Fraktal'nyj analiz finansovykh rynkov. Primenenie teorii khaosa v investitsiyakh i ehkonomike (Fractal analysis of financial markets. Application of chaos theory to investments and Economics). M.: Internet trading, 2004. $304 \mathrm{p}$.

3. Mandelbrot R. L., Hadson R. L. (Ne)poslushnye rynki. Fraktal'naya revolyutsiya v finansakh (Hudson (not) obedient markets. Fractal revolution in Finance). M.: Williams, 2006. 408 p.

4. Peters E. KHaos i poryadok na rynkakh kapitala (Chaos and order in capital markets). M.: World, 2000. 333 p

5. Fraktal'naya teoriya rynka Forex (The Fractal theory of the Forex market). M.: Admiral Markets, 2009. $296 \mathrm{p}$.

\section{СВЕДЕНИЯ ОБ АВТОРАХ}

Чикатуева Диана Эдуардовна, студентка 4 курса бакалавриата, направление подготовки 38.03 .05 «Бизнес-информатика», СКФУ. E-mail: diana.chikatueva@mail.ru

Сильченко Алина Сергеевна, студентка 4 курса бакалавриата, направление подготовки 38.03 .05 «Бизнес-информатика», СКФУ. E-mail: alya.silchenko.97@mail.ru

Рачкова Татьяна Геннадиевна, студентка 4 курса бакалавриата, направление подготовки 38.03 .05 «Бизнес-информатика», СКФУ. E-mail: tania.260198@mail.ru

Оробец Кирилл Геннадьевич, студент 3 курса магистратуры, направление подготовки 38.04 .05 «Бизнес-информатика», СКФУ. E-mail: grv222@mail.ru

\section{INFORMATION ABOUT AUTHORS}

Diana Chikatueva, 4th year undergraduate student, training direction 38.03.05 «Business Informatics», NCFU. E-mail: diana.chikatueva $a$ mail.ru

Alina Silchenko, 4th year undergraduate student, direction of training 38.03.05 «Business Informatics». NCFU. E-mail: alya.silchenko.97@mail.ru

Tatyana Rachkova, 4th year undergraduate student, direction of training 38.03 .05 «Business Informatics», NCFU. E-mail: tania.260198@mail.ru

Kirill Orobets, 3rd year undergraduate student, direction of training 38.04.05 «Business Informatics», NCFU. E-mail: grv222@mail.ru 